\title{
Evaluation of a semi-automated in vitro feeding system for Dermacentor reticulatus and Ixodes ricinus adults
}

\author{
Bettina Böhme $^{1} \cdot$ Christoph Krull $^{1} \cdot$ Peter-Henning Clausen $^{1} \cdot$ Ard M. Nijhof ${ }^{1}$
}

Received: 13 July 2017 / Accepted: 9 October 2017 /Published online: 3 January 2018

(C) The Author(s) 2018. This article is an open access publication

\begin{abstract}
The long feeding duration of ixodid ticks and need for regular blood changes turns the artificial feeding of ticks into a tedious process. To reduce the number of blood changes, a semi-automated system (SAS) for the artificial feeding of hard ticks was developed and evaluated. It consisted of a glass feeding reservoir that can accommodate six tick feeding chambers. A peristaltic pump was used to pump blood through the feeding reservoir, which was changed once daily. Groups of Dermacentor reticulatus and Ixodes ricinus adults were fed simultaneously in both the SAS and a conventional in vitro feeding system. In the conventional system, feeding chambers were hung inside a glass beaker filled with blood that was replaced twice daily. Dermacentor reticulatus adults fed in the SAS obtained significantly higher engorgement weights. Although engorgement rates between both systems were comparable, significantly more SAS-fed females laid fertile egg batches. The egg batch weight of SAS-fed females was also significantly higher. In contrast, the engorgement rate and fecundity of SAS-fed I. ricinus were significantly reduced in comparison to ticks fed in the conventional system. This reduction was likely to be caused by fungal infestation, which could spread between feeding chambers in the SAS. Although the SAS reduced the workload compared to the conventional feeding system and showed promising results for the in vitro feeding of $D$. reticulatus adults, measures to prevent fungal infestations in the SAS should be considered in future studies.
\end{abstract}

Bettina Böhme and Christoph Krull contributed equally to this work

Ard M. Nijhof

ard.nijhof@fu-berlin.de

1 Institute for Parasitology and Tropical Veterinary Medicine, Freie Universität Berlin, Robert-von-Ostertag-Str. 7-13,

14163 Berlin, Germany
Keywords Ixodes ricinus · Dermacentor reticulatus . Artificial tick feeding

\section{Introduction}

Ticks are obligate haematophagous ectoparasites that infest both humans and animals and may act as vectors of pathogens. Two common tick species in Central Europe are Ixodes ricinus (Linné, 1758) and Dermacentor reticulatus (Fabricius, 1794). I. ricinus is the most widespread tick in Central and Western Europe. It has a broad host range and can transmit a wide variety of pathogens, including Borrelia burgdorferi sensu lato causing Lyme borreliosis, Anaplasma phagocytophilum causing human granulocytic anaplasmosis, Babesia microti, Babesia venatorum and Babesia divergens causing babesiosis in humans and animals and tick-borne encephalitis virus (Jongejan and Uilenberg 2004). Dermacentor reticulatus can be found in many parts of Europe, and its distribution in many regions has expanded during the last decades (Rubel et al. 2016). Its veterinary relevance lies in its role as a vector for Babesia canis and Theileria equi, causing babesiosis in dogs and horses, respectively, but it has also been associated with the transmission of human pathogens such as Rickettsia slovaca and Rickettsia raoultii, causing tick-borne lymphadenopathy (TIBOLA), and a flavivirus causing Omsk haemorrhagic fever (Jongejan and Uilenberg 2004).

For research on ticks and tick-borne pathogens, the use of live ticks is often required, and tick colonies are routinely maintained in laboratories worldwide (Levin and Schumacher 2016). All life stages are usually fed on experimental animals, which most closely resemble the natural situation and is highly efficient, but is also associated with ethical concerns. An alternative to the feeding of ticks on animals is the artificial feeding of ticks, which provides interesting potential research applications and also 
adheres to the $3 \mathrm{R}$ principles to 'Reduce, Replace and Refine' the use of experimental animals in research (Russel and Burch 1959). However, artificial tick feeding is associated with drawbacks that have restricted their application, as not all tick life stages and species, in particular those with shorter mouthparts, can be fed successfully in vitro, and a significant reduction in tick fertility has been reported for artificially fed ticks. Finally, artificial tick feeding requires a blood donor, expertise and considerable manual labour (Kuhnert 1996; Krull et al. 2017).

Artificial tick feeding usually requires a synthetic membrane or animal skin, through which the ticks feed on a provided blood source. The blood used for in vitro feeding needs to be constantly heated to the host body temperature to accommodate tick feeding (Voigt et al. 1993), which at the same time provides optimal conditions for bacterial and fungal growth and decay of the blood meal when blood collection and/or in vitro tick feeding are performed under unsterile conditions. To prevent this from happening, the addition of broad-spectrum antibiotics and fungicides to the blood and a regular change of the blood meal, typically twice daily but up to six times per day, are commonly employed for in vitro tick feeding (Waladde et al. 1993; Kuhnert et al. 1995; Krober and Guerin 2007). As tick feeding may take up to several weeks (Jones et al. 1988), the manual labour associated with artificial tick feeding is considerable.

An attempt to partially automate the feeding of haematophagous arthropods to reduce the associated workload of manual blood changes has been described (Kuhnert et al. 1998). Although one of the experiments performed using this system resulted in engorgement weights and fertility rates that were not significantly different from Amblyomma hebraeum adults fed in vivo on cattle, the number of ticks that could be fed was limited and the approach was not pursued further. Another interesting artificial feeding approach was published for colony maintenance of the human head louse, Pediculus capitis. In this system, blood and saline flowed alternately through a heated feeding reservoir on which up to 13 small feeders with a diameter of $1.25 \mathrm{~cm}$ could be placed (Takano-Lee et al. 2003).

In this study, the use of a novel semi-automated artificial tick feeding system (SAS) was evaluated for feeding D. reticulatus and I. ricinus adults. The SAS uses the same glass feeding units as previously used for a study on the optimization of in vitro feeding for D. reticulatus (Krull et al. 2017). By performing the tick feedings simultaneously in both SAS and this conventional system, we were able to compare the feeding success and fertility of the ticks between both systems.

\section{Material and methods}

\section{Ticks}

Adult $I$. ricinus and D. reticulatus ticks were collected by flagging the vegetation of fallow land and forests in and near
Berlin. Ticks were stored in glass containers with pierced lids in a desiccator containing saturated $\mathrm{MgSO}_{4}$ solution, providing a relative humidity of approximately $90 \%$ at room temperature and a 15:9-h light/dark cycle.

To evaluate the efficacy of the SAS in comparison to the conventional system, adult ticks were randomly placed in two groups for subsequent artificial feeding. Artificial feeding experiments in the SAS and the conventional system were performed simultaneously for each species. The D. reticulatus experiments were performed in 2014 and the I. ricinus experiments in 2016. The bovine blood used in both systems within a single experiment originated from the same animal. For D. reticulatus, seven female ticks were used per feeding chamber and two experiments with six feeding chambers for each system were performed, resulting in a total number of 84 females for each tested system. For I. ricinus, ten females were used per feeding unit in the first experiment, and nine females per feeding unit were used in the second experiment, which resulted in a total number of 114 females per system tested.

\section{Conventional artificial tick feeding system}

Preparation of blood, tick feeding units and the blood change procedure was performed as previously described (Krull et al. 2017). Feeding units with $I$. ricinus were kept in an incubator (ICH256C, Memmert, Düsseldorf, Germany) at $20{ }^{\circ} \mathrm{C}, 80 \%$ RH, $2.5 \% \mathrm{CO}_{2}$ and a 15:9-h light/dark cycle. For logistical reasons, the feeding of $D$. reticulatus was performed in a waterbath set at $38^{\circ} \mathrm{C}$ and a $15: 9$-h light/dark cycle. Additional $\mathrm{CO}_{2}$ was not provided for the $D$. reticulatus feeding experiments.

\section{Semi-automated feeding system}

The SAS consisted of a custom-made hexagonal borosilicate glass feeding reservoir (Technische Universität Berlin, Germany) which featured one outlet of $5 \mathrm{~mm}$ diameter on both ends (Fig. 1). The feeding reservoir was covered by a separate hexagonal glass plate containing six holes of $40 \mathrm{~mm}$ diameter, with a minimum distance of $20 \mathrm{~mm}$ between the holes. A single glass feeding unit, identical to the one used in the conventional system, could be hung in each hole using a rubber ring with an inner diameter of $32 \mathrm{~mm}$ (Lux, Wermelskirchen, Germany) (Fig. 2).

The blood reservoir consisted of a 1-L bottle which contained $600 \mathrm{~mL}$ blood supplemented with heparin (20 IU/ mL, Ratiopharm, Ulm, Germany), glucose (2 g/L blood, Carl Roth, Karlsruhe, Germany), adenosine triphosphate (ATP) (51 mg/mL, Carl Roth) and gentamycin (Carl Roth) $(5 \mu \mathrm{g} /$ $\mathrm{mL})$. Gentamycin and ATP were added to the blood reservoir just before use. The blood reservoir was placed inside a Styrofoam box and refrigerated with frozen ice packs that were changed daily. Blood was pumped from the bottle using a peristaltic pump (Reglo ICC, Ismatec, Wertheim, Germany) 
Fig. 1 Feeding reservoir with inserted tick feeding units. Membranes, tubing and lids are not shown. $A=270 \mathrm{~mm}$, $\mathrm{B}=125 \mathrm{~mm}, \mathrm{C}=30 \mathrm{~mm}$

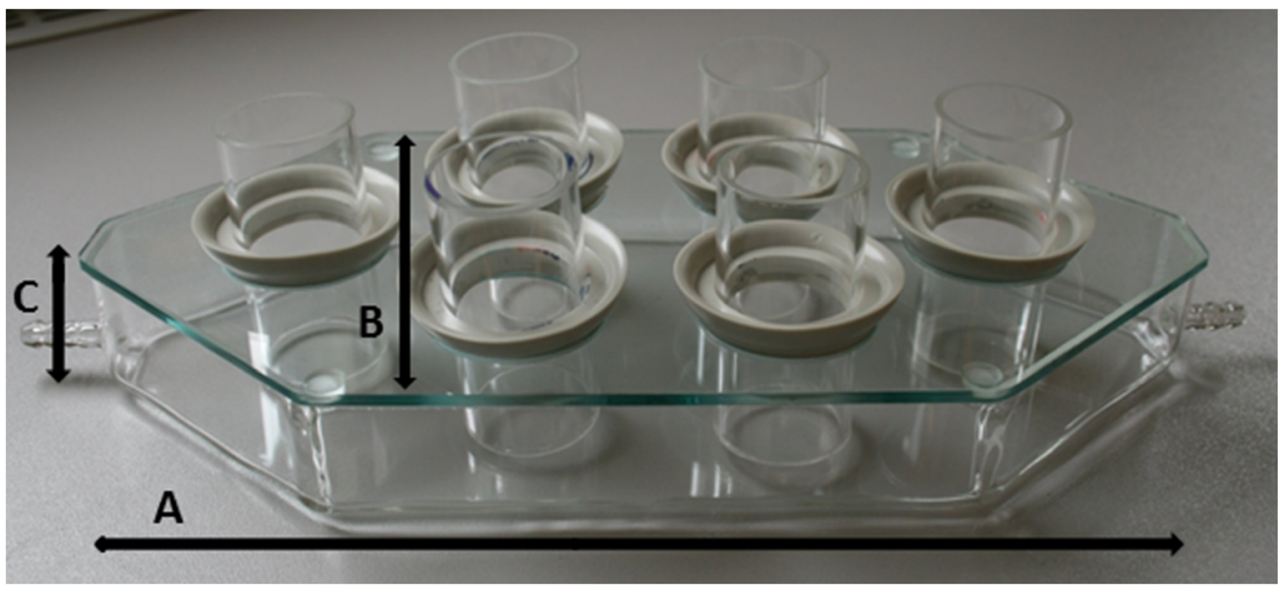

through silicone tubing into the feeding reservoir at a rate of $0.2 \mathrm{~mL} / \mathrm{min}$. A second tube with a $0.2-\mu \mathrm{m}$ syringe filter (Carl Roth) was connected to the bottle cap for pressure equalisation. The outlet of blood from the feeding reservoir occurred passively through a silicon tube which led to a receptacle containing the waste blood that was discarded. The blood meal was heated to $37{ }^{\circ} \mathrm{C}$ by placing the feeding reservoir on top of a heating plate (Hot Plate 062, Labotect, Göttingen, Germany). Dermacentor reticulatus feeding was performed in the laboratory at a room temperature of $20{ }^{\circ} \mathrm{C}$ and a 15:9-h light/dark cycle without additional $\mathrm{CO}_{2}$. Damp cotton balls on top of the tick chambers provided the ticks with a RH of approx. $70 \%$. All feeding experiments for I. ricinus were performed in an incubator (ICH256C, Memmert) at $20{ }^{\circ} \mathrm{C}, 80 \% \mathrm{RH}, 2.5 \% \mathrm{CO}_{2}$ and a $15: 9$-h light/dark cycle. The slow influx of refrigerated blood into the heated flowthrough chamber ensured a constant temperature. Tick chambers were hung into the holes of the glass plate covering the feeding reservoir so that the underside of the feeding chambers was immersed in blood. The environmental conditions inside the feeding chambers for the second D. reticulatus experiment and both I. ricinus experiments were recorded by an iButton (Hygrochron, Maxim Integrated, San Jose, USA) that measured temperature and RH at 30-min intervals.

Blood change for the SAS was done once per day. The feeding reservoir was disconnected and placed on a heating plate (Labotect) in the laboratory. A new sterile feeding reservoir was connected to the pump and filled with fresh blood at a rate of $30 \mathrm{~mL} / \mathrm{min}$. The blood was left to be warmed whilst the feeding units were examined for detached ticks and membrane integrity and rinsed with pre-heated sterile $0.9 \% \mathrm{NaCl}$ solution. The rinsed feeding units were placed in a new sterile feeding reservoir lid which was placed on top of the feeding reservoir. Every second day, the blood reservoir was depleted
Fig. 2 Schematic drawing of the semi-automated artificial tick feeding system. ID inner diameter, $\mathrm{AD}$ outer diameter of tubing

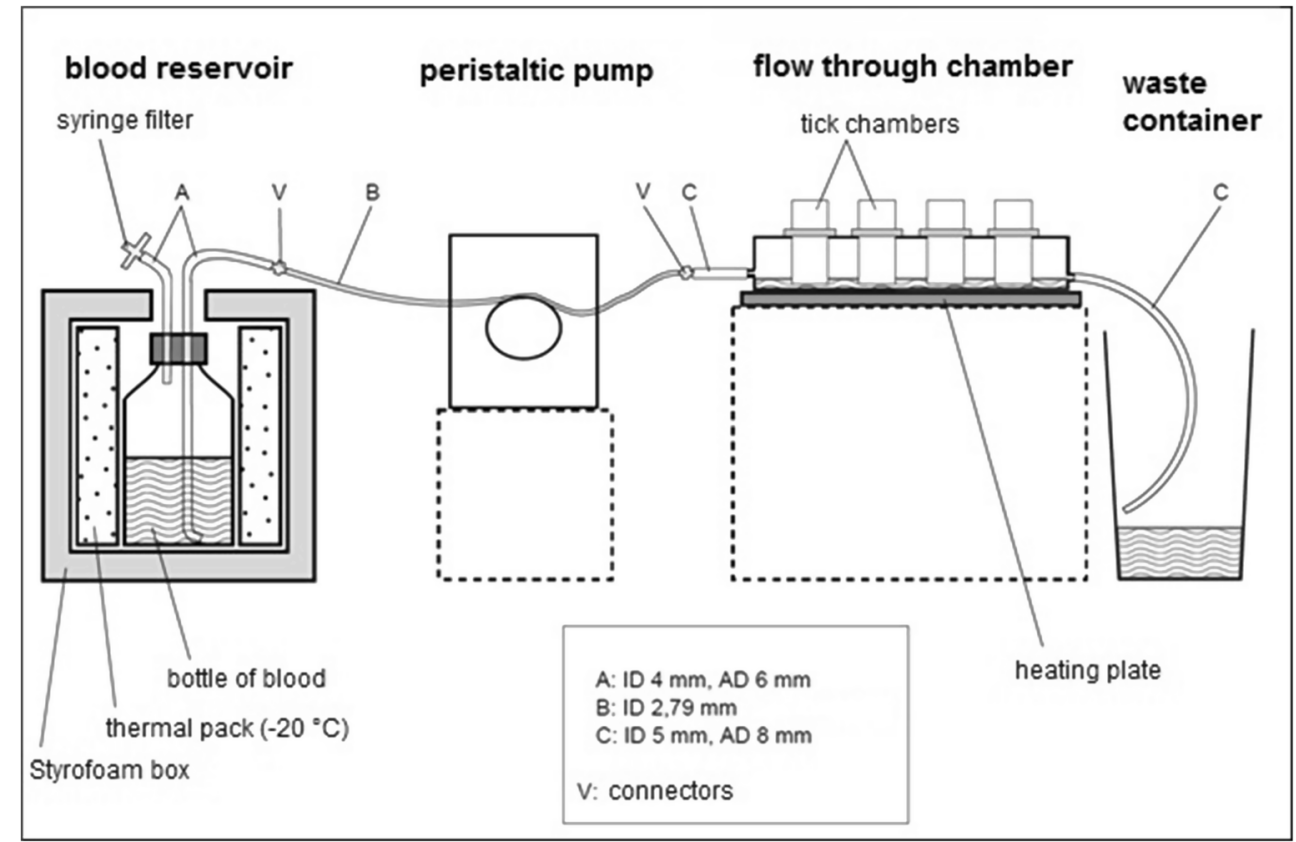


and replaced with a new bottle and new tubing. The blood reservoir bottles, silicon tubing, feeding reservoirs and glass plates covering the feeding reservoir were washed and autoclaved before use.

\section{Statistical analysis}

Statistical analyses were performed using SPSS software. Engorgement rates and rates of females producing fertile eggs were evaluated using Fisher's exact test. Engorgement masses and egg batch masses were evaluated using $t$ test or MannWhitney $U$ test, depending on normal distribution of the data.

\section{Results}

\section{Dermacentor reticulatus}

In the conventional system, $26.2 \%$ females engorged (22/84), which was not significantly different from the number of ticks which engorged in the SAS $(27.4 \%, 23 / 84, p=1.00$, Fisher's exact test). The average mass of engorged females differed significantly between both systems, with $185.2 \pm 78.2 \mathrm{mg}$ in the conventional group and $269.6 \pm 105.1 \mathrm{mg}$ in the SAS group ( $p=0.004, t$ test). The number of engorged females producing fertile egg batches differed significantly: $22.7 \%$ $(5 / 22)$ in the conventional group and $87.0 \%(20 / 23)$ in the SAS group $(p<0.0001$, Fisher's exact test). The mass of the egg batches was also significantly different between both groups $(80.0 \pm 37.8 \mathrm{mg}$ vs. $123.0 \pm 71.4 \mathrm{mg}, p=0.031, t$ test $)$. The average feeding duration, defined here as the time between placement of the tick in the feeding chamber and its detachment as an engorged specimen from the membrane, was $10 \pm 3$ days in the conventional group and $10 \pm 2$ days in the SAS group (Table 1). iButton data from the second experiment showed small differences in the microclimate inside the tick feeding unit. The $\mathrm{RH}$ and temperature were
$78.0 \pm 2.4 \%$ and $38.1 \pm 0.2{ }^{\circ} \mathrm{C}$ in the conventional system and $74.2 \pm 3.0 \%$ and $36.4 \pm 0.4{ }^{\circ} \mathrm{C}$ in the SAS.

\section{Ixodes ricinus}

iButton data showed that the RH inside the tick feeding units averaged to $61 \pm 9 \%$ in the conventional system and to $54 \pm 10 \%$ in the SAS, and thus remained lower than the set incubator RH of $80 \%$. Of the ticks fed in the conventional system, $80.7 \%$ engorged $(92 / 114)$. This was significantly more than the number of engorged ticks in the SAS system, where $20.2 \%(23 / 114, p<0.0001$, Fisher's exact test $)$ engorged. The average mass of engorged females was not significantly different, with $217.0 \pm 95.6 \mathrm{mg}$ in the conventional system and $204.5 \pm 131.6 \mathrm{mg}$ in the SAS $(p=0.218$, Mann-Whitney $U$ test). The number of engorged females producing fertile egg batches differed significantly: $66.3 \%$ (61/ $92)$ in the conventional system and $30.4 \%(7 / 23)$ in the SAS ( $p=0.0037$, Fisher's exact test). No significant difference could be found between the deposited egg mass of $56.5 \pm 33.4 \mathrm{mg}$ by females fed in the conventional system and $53.9 \pm 30.4 \mathrm{mg}$ by females fed in the SAS $(p=0.984$, Mann-Whitney $U$ test). The average feeding duration was $11 \pm 2$ days in the conventional group and $11 \pm 2$ days in the SAS group. A noticeable difference was fungal infection, which affected five of six feeding units in the SAS in the first experiment and four of six feeding units in the second experiment, whereas fungal infections were not observed in the conventional system. Of the seven SAS-fed ticks that laid fertile egg batches, six were fed in feeding units that were not affected by fungi.

\section{Discussion}

We previously demonstrated that the in vitro feeding of D. reticulatus adults could significantly be improved by

Table 1 Effects of in vitro feeding using the conventional and semi-automated feeding system (SAS) on the engorgement and fecundity of Dermacentor reticulatus and Ixodes ricinus females

\begin{tabular}{|c|c|c|c|c|c|c|}
\hline Species & $\begin{array}{l}\text { Experimental } \\
\text { group }\end{array}$ & $\begin{array}{l}\text { Engorgement } \\
\text { rate }\end{array}$ & $\begin{array}{l}\text { Engorgement } \\
\text { mass } \pm \mathrm{SD}(\mathrm{mg})\end{array}$ & $\begin{array}{l}\text { Females producing } \\
\text { fertile eggs }\end{array}$ & $\begin{array}{l}\text { Egg batch } \\
\text { mass } \pm \text { SD (mg) }\end{array}$ & $\begin{array}{l}\text { Time to repletion } \\
\text { (day) }\end{array}$ \\
\hline \multirow[t]{2}{*}{ D. reticulatus } & Conventional & $26.2 \%(22 / 84)$ & $185.2 \pm 78.2^{b}$ & $22.7 \%(5 / 22)^{\mathrm{c}}$ & $80.0 \pm 37.8^{\mathrm{e}}$ & $10.1 \pm 2.5$ \\
\hline & SAS & $27.4 \%(23 / 84)$ & $269.6 \pm 105.1^{\mathrm{b}}$ & $87.0 \%(20 / 23)^{\mathrm{c}}$ & $123.0 \pm 71.4^{\mathrm{e}}$ & $9.8 \pm 2.1$ \\
\hline \multirow[t]{2}{*}{ I. ricinus } & Conventional & $80.7 \%(92 / 114)^{\mathrm{a}}$ & $217.0 \pm 95.6$ & $66.3 \%(61 / 92)^{\mathrm{d}}$ & $56.5 \pm 33.4$ & $10.8 \pm 2.2$ \\
\hline & SAS & $20.2 \%(23 / 114)^{\mathrm{a}}$ & $204.5 \pm 131.6$ & $30.4 \%(7 / 23)^{\mathrm{d}}$ & $53.9 \pm 30.4$ & $11.4 \pm 1.6$ \\
\hline
\end{tabular}

\footnotetext{
${ }^{a}$ Significant difference (Fisher's exact test, $p<0.0001$ )

${ }^{\mathrm{b}}$ Significant difference $(t$ test, $p=0.004)$

${ }^{\mathrm{c}}$ Significant difference (Fisher's exact test, $p<0.0001$ )

${ }^{\mathrm{d}}$ Significant difference (Fisher's exact test, $p=0.0037$ )

${ }^{\mathrm{e}}$ Significant difference ( $t$ test, $p=0.031$ )
} 
exposing ticks to increased $\mathrm{CO}_{2}$ levels during feeding and by adding extra glucose to the blood meal (Krull et al. 2017). In the study presented here, we focused on the evaluation of a system that would significantly reduce the labour associated with in vitro tick feeding, whilst maintaining desirable feeding and reproduction results. This SAS was considered to be semiautomated, since a change of the feeding reservoir once per day was necessary to prevent the accumulation of blood coagulations inside the reservoir. Nonetheless, the workload could be reduced by half compared to the conventional manual feeding system in which the blood-filled beakers were changed twice daily. A drawback of the SAS is the larger volume of blood required due to the steady flow of blood through the system. In the setup presented here, a reservoir of $600 \mathrm{~mL}$ provided six feeding chambers in the SAS with blood for a period of $48 \mathrm{~h}$. In the conventional system and within the same period, six feeding chambers required $120 \mathrm{~mL}$ blood, i.e., five times less than the SAS. The use of the SAS for the artificial feeding of ticks would therefore be of most interest in situations where the blood supply is not a limiting factor. We refrained from the re-use of blood through a conversion of the SAS into a circulatory system, as this was considered likely to result in obstructions of the tubing caused by blood coagula and facilitate the further growth of any contaminants present.

The SAS was most successful for the feeding of $D$. reticulatus adults, as these obtained a significantly higher engorgement weight and laid more fertile eggs than their counterparts fed on the same blood source in the conventional system. Ticks gain most of their weight during the rapid phase of engorgement, which usually lasts 1-2 days on a natural host and is associated with enhanced salivation and increased blood flow to the tick bite site (Tatchell et al. 1972; Fielden et al. 1999). Secreted tick saliva is more likely to disperse in the SAS due to the steady stream of blood below the membrane compared to the conventional system. This enhanced dispersal might attribute to the increased weight gain of D. reticulatus ticks observed in the SAS, as it more closely mimics the natural situation. Increased concentrations of salivary components around the mouthparts of ticks feeding in the more stagnant conventional system might have inhibited further feeding (Kemp et al. 1982) and the uptake of nutritional blood constituents, leading to a suboptimal weight gain and lower engorgement weights. Although small differences in $\mathrm{RH}$ and temperature inside the tick feeding units between both systems were observed, we consider it unlikely that these differences explain the differences in engorgement weight and fecundity. The artificial feeding of Rhipicephalus sanguineus at 27 and $37^{\circ} \mathrm{C}$, a far greater temperature difference than we observed, was recently shown to have no significant effect on the mean weight gain of ticks (Valim et al. 2017).

The engorgement rates of $D$. reticulatus females fed in this study were relatively low for both the conventional and SAS method (26.2-27.4\%), whereas the engorgement rate of I. ricinus females fed using the conventional in vitro system $(80.7 \%)$ was much higher. The precise reasons for this are unknown, but features of the in vitro system such as membrane structure and attachment stimuli used may have played a role. It should also be noted that $I$. ricinus is a prostriate tick; all females had ample access to males prior to their introduction in the tick feeding chambers and it is likely that copulation had already taken place, obviating mating during feeding (Kiszewski et al. 2001) which might have facilitated the in vitro engorgement for this species.

Although we did not observe significant differences of the feeding duration for I. ricinus between the conventional in vitro fed group and the SAS fed group, the duration was longer than the 6-8 days, which $I$. ricinus typically needs to engorge on cattle (data not shown). Other groups have also reported a longer duration of tick feeding in vitro compared to ticks fed on animals (Kuhnert et al. 1995; Liu et al. 2014). We observed that some ticks placed in the tick feeding chamber did not attach immediately, but needed several days until attachment and feeding took place. This may in part explain the longer in vitro feeding duration observed here. Encouragement of questing behaviour and swift tick attachment through the provision of additional stimuli such as $\mathrm{CO}_{2}$, host or synthetic odours, visual cues, vibrations and membranes that more closely mimic the natural hosts' skin could shorten the feeding duration and further improve the artificial feeding of ticks (Waladde et al. 1996; Krull et al. 2017; Trentelman et al. 2017).

The most plausible explanation for the reduced feeding success and fecundity of I. ricinus in the SAS were the severe fungal infestations of the membranes, which affected 9 of 12 feeding chambers. It is likely that spread of the fungal contamination between the different feeding chambers was facilitated by the flow of blood within the SAS. The origin of the contamination remained unclear, but blood or ticks were possible sources. Bovine blood was collected during exsanguination at a slaughterhouse and contact with the natural mycoflora of cattle hair and skin could not be excluded.

Ticks, including $I$. ricinus and $D$. reticulatus, have been reported to harbour various species of fungi (Samsinakova et al. 1974; Bell 1980; Kalsbeek et al. 1995). Kuhnert observed that fungal growth on the artificial feeding membranes started predominantly on cement cones of tick feeding sites and identified these fungi as Aspergillus fumigatus and A. ochraeus (Kuhnert 1995). Although measures were taken to minimise the risk of contamination during the in vitro feeding process in the laboratory, an environmental contamination cannot be fully excluded either. Surface sterilisation of ticks prior to artificial feeding, or the addition of fungicides to the blood meal may solve this problem. Since treatment of ticks with antibiotics may have a negative impact on tick fitness and fecundity (Zhong et al. 2007; Narasimhan et al. 2014) and 
potentially symbiotic fungi have been reported from Ixodes ticks (Benoit et al. 2005), the addition of biocidal products to the blood meal may have negative consequences for the ticks, which should be carefully assessed.

In conclusion, the SAS evaluated in this study showed promising results for the feeding of $D$. reticulatus adults. Dermacentor reticulatus ticks fed using the SAS obtained significantly higher engorgement weights and reproduction rates compared to ticks fed in vitro using the conventional system. In addition, the SAS required less manual work. By contrast, the poor feeding results for I. ricinus and increased fungal contamination in the SAS found when feeding this tick species also revealed a weakness of the SAS. The prevention of fungal contamination in the SAS should have priority in further studies to examine its suitability for feeding additional tick species.

Acknowledgements The authors would like to thank the staff of the abattoir where the blood was collected and Mrs. Peggy Hoffmann-Köhler for her technical assistance.

Funding Information This project was funded by the German Federal Ministry of Education and Research (BMBF, grant 031A228) as part of its 'Alternative methods to animal testing' programme.

Open Access This article is distributed under the terms of the Creative Commons Attribution 4.0 International License (http:// creativecommons.org/licenses/by/4.0/), which permits unrestricted use, distribution, and reproduction in any medium, provided you give appropriate credit to the original author(s) and the source, provide a link to the Creative Commons license, and indicate if changes were made.

\section{References}

Bell LJ (1980) Organ culture of Rhipicephalus appendiculatus with maturation of Theileria parva in tick salivary glands in vitro. Acta Trop 37(4):319-325

Benoit JB, Yoder JA, Ark JT, Rellinger EJ (2005) Fungal fauna of Ixodes scapularis say and Rhipicephalus sanguineus (Latreille) (Acari : Ixodida) with special reference to species-associated internal mycoflora. Int J Acarol 31(4):417-422

Fielden LJ, Jones RM, Goldberg M, Rechav Y (1999) Feeding and respiratory gas exchange in the American dog tick, Dermacentor variabilis. J Insect Physiol 45(4):297-304

Jones LD, Davies CR, Steele GM, Nuttall PA (1988) The rearing and maintenance of ixodid and argasid ticks in the laboratory. Animal Technol 39(2):99-106

Jongejan F, Uilenberg G (2004) The global importance of ticks. Parasitology 129(Suppl):S3-14

Kalsbeek V, Frandsen F, Steenberg T (1995) Entomopathogenic fungi associated with Ixodes ricinus ticks. Exp Appl Acarol 19(1):45-51

Kemp DH, Stone BF, Binnington KC (1982) Tick attachment and feeding: role of the mouthparts, feeding apparatus, salivary gland secretions and the host response. In: Obenchain FD, Galun R (eds) Physiology of ticks, vol 1. Pergamon Press, Oxford, pp 119-168

Kiszewski AE, Matuschka FR, Spielman A (2001) Mating strategies and spermiogenesis in ixodid ticks. Annu Rev Entomol 46:167-182
Krober T, Guerin PM (2007) An in vitro feeding assay to test acaricides for control of hard ticks. Pest Manag Sci 63(1):17-22

Krull C, Bohme B, Clausen PH, Nijhof AM (2017) Optimization of an artificial tick feeding assay for Dermacentor reticulatus. Parasit Vectors 10(1):60

Kuhnert F (1995) Untersuchungen zur in vitro Zucht von Schildzecken (Acari: Ixodidae). Cuvillier, Göttingen

Kuhnert F (1996) Feeding of hard ticks in vitro: new perspectives for rearing and for the identification of systemic acaricides. ALTEX 13(2):76-87

Kuhnert F, Diehl PA, Guerin PM (1995) The life-cycle of the bont tick Amblyomma hebraeum in vitro. Int J Parasitol 25(8):887-896

Kuhnert F, Issmer AE, Grunewald J (1998) Teilautomatisierte in vitro Fütterung adulter Schildzecken (Amblyomma hebraeum). ALTEX 15(2):67-72

Levin ML, Schumacher LB (2016) Manual for maintenance of multi-host ixodid ticks in the laboratory. Exp Appl Acarol 70(3):343-367

Liu XY, Cote M, Paul RE, Bonnet SI (2014) Impact of feeding system and infection status of the blood meal on Ixodes ricinus feeding. Ticks Tick Borne Dis 5(3):323-328

Narasimhan S, Rajeevan N, Liu L, Zhao YO, Heisig J, Pan JY, EpplerEpstein R, DePonte K, Fish D, Fikrig E (2014) Gut microbiota of the tick vector Ixodes scapularis modulate colonization of the Lyme disease spirochete. Cell Host Microbe 15(1):58-71

Rubel F, Brugger K, Pfeffer M, Chitimia-Dobler L, Didyk YM, Leverenz S, Dautel H, Kahl O (2016) Geographical distribution of Dermacentor marginatus and Dermacentor reticulatus in Europe. Ticks Tick Borne Dis 7(1):224-233

Russel WMS, Burch RL (1959) The principles of humane experimental technique. Methuen, London

Samsinakova A, Kalalova S, Daniel M, Dusbabek F, Honzakova E, Cerny V (1974) Entomogenous fungi associated with the tick Ixodes ricinus (L.) Folia Parasitol (Praha) 21(1):39-48

Takano-Lee M, Velten RK, Edman JD, Mullens BA, Clark JM (2003) An automated feeding apparatus for in vitro maintenance of the human head louse, Pediculus capitis (Anoplura: Pediculidae). J Med Entomol 40(6):795-799

Tatchell RJ, Carnell R, Kemp DH (1972) Electrical studies on the feeding of the cattle-tick, Boophilus microplus. Z Parasitenkd 38(1):32-44

Trentelman JJ, Kleuskens JA, van de Crommert J, Schetters TP (2017) A new method for in vitro feeding of Rhipicephalus australis (formerly Rhipicephalus microplus) larvae: a valuable tool for tick vaccine development. Parasit Vectors 10(1):153

Valim JR, Rangel CP, Baeta BA, Ribeiro CC, Cordeiro MD, Teixeira RC, Cepeda PB, Fonseca AH (2017) Using plastic tips in artificial feeding of Rhipicephalus sanguineus sensu lato (Acari: Ixodidae) females. Rev Bras Parasitol Vet 26(1):110-114

Voigt WP, Young AS, Mwaura SN, Nyaga SG, Njihia GM, Mwakima FN, Morzaria SP (1993) In vitro feeding of instars of the ixodid tick Amblyomma variegatum on skin membranes and its application to the transmission of Theileria mutans and Cowdria ruminantum. Parasitology 107(3):257-263

Waladde SM, Young AS, Morzaria SP (1996) Artificial feeding of ixodid ticks. Parasitol Today 12(7):272-278

Waladde SM, Young AS, Ochieng SA, Mwaura SN, Mwakima FN (1993) Transmission of Theileria parva to cattle by Rhipicephalus appendiculatus adults fed as nymphae in vitro on infected blood through an artificial membrane. Parasitology 107(3):249-256

Zhong J, Jasinskas A, Barbour AG (2007) Antibiotic treatment of the tick vector Amblyomma americanum reduced reproductive fitness. PLoS One 2(5):e405 